\title{
Aspectos de saúde coletiva e ocupacional associados à gestão dos resíduos sólidos municipais
}

\author{
Public and occupational health issues related \\ to municipal solid waste management
}

João Alberto Ferreira 1

Luiz Antonio dos Anjos 2,3

\footnotetext{
1 Departamento de Engenharia Sanitária e do Meio Ambiente, Faculdade de Engenharia, Universidade do Estado do Rio de Janeiro. Rua São Francisco Xavier 524, bloco A, sala 5002, Rio de Janeiro, $R J$ 20550-013, Brasil. joaf@ruralrj.com.br

2 Laboratório de Avaliação Nutricional e Funcional, Departamento de Nutrição Social, Faculdade de Nutrição, Universidade Federal Fluminense. Rua São Paulo 30, 4o andar Niterói, RJ 24040-110, Brasil. 3 Centro de Estudos da Saúde do Trabalhador e Ecologia Humana, Escola Nacional de Saúde Pública, Fundação Oswaldo Cruz. Rua Leopoldo Bulhões 1480 Rio de Janeiro, $R J$ 21041-210, Brasil. anjos@ensp.fiocruz.br
}

\begin{abstract}
Based on a literature review, the paper calls attention to public and occupational health problems related to poor municipal solid waste management resulting from a development model that views environmental protection, public health, and workers' health as secondary issues. The authors emphasize the need for research to support a new waste management model highlighting the importance of environmental preservation and protection of human health, in addition to suggesting measures to achieve such goals. The discussion aims to incorporate solid waste management into the public health agenda.
\end{abstract}

Key words Occupational Health; Solid Wastes; Urban Cleaning

Resumo O artigo objetiva despertar a atenção para os problemas de saúde coletiva e saúde ocupacional associados aos resíduos sólidos municipais, particularmente, em função da má gestão dos mesmos e de um modelo de desenvolvimento no qual o meio ambiente, a saúde pública e a saúde do trabalhador são relegados a um plano secundário. Escrito com base em uma revisão bibliográfica, o artigo foi enriquecido por alguns resultados de estudos recentes dos quais os autores participaram. São apontados alguns eixos principais da questão que ajudam a compreender a ausência, quase total, de estudos e pesquisas que possam embasar uma gestão dos resíduos, que considere a preservação do meio ambiente e da saúde humana. Ao procurar ampliar a discussão sobre os resíduos sólidos o que se busca é a sua inserção, de forma mais significativa, como tema da saúde pública.

Palavras-chave Saúde Ocupacional; Resíduos Sólidos; Limpeza Urbana 


\section{Introdução}

Embora não existam dúvidas sobre a importância da atividade de limpeza urbana para o meio ambiente e para a saúde da comunidade, esta percepção não se tem traduzido em ações efetivas que possibilitem mudanças qualitativas na situação negativa em que se encontram, de forma geral, os sistemas de gerenciamento de resíduos sólidos urbanos em toda a América Latina, inclusive no Brasil.

Os efeitos adversos dos resíduos sólidos municipais no meio ambiente, na saúde coletiva e na saúde do indivíduo são reconhecidos por diversos autores (Accurio et al., 1998; Anjos et al., 1995; Cantanhede, 1997; Diaz et al., 1997; Ferreira, 1997; Leite \& Lopes, 2000; Maglio, 2000; Robazzi et al., 1992; Velloso, 1995; Zepeda, 1995), que apontam as deficiências nos sistemas de coleta e disposição final e a ausência de uma política de proteção à saúde do trabalhador, como os principais fatores geradores desses efeitos.

Apesar desse reconhecimento, são escassos os estudos e pesquisas realizados no Brasil e na América Latina sobre o assunto. Isto se dá, em parte, pelo fato de existirem poucos centros de pesquisas que tratam das questões dos resíduos sólidos municipais e, na maioria das vezes, os trabalhos não incorporarem, a não ser em raras ocasiões, os componentes saúde e meio ambiente. Alguns dos fatores que contribuem, em maior ou menor grau, para esta situação são:

- O pouco interesse que os resíduos comuns, do cotidiano, despertam nos profissionais e pesquisadores, especialmente entre aqueles com formação em países desenvolvidos, onde as questões e o nível de conhecimento sobre estes resíduos são relacionados a outro estágio de desenvolvimento. Também contribui para esta situação, a pequena pressão exercida pela população - desde que haja uma coleta domiciliar - para os problemas decorrentes da gestão dos resíduos sólidos;

- A pouca atenção dada pelo poder público às questões de saúde em geral e que repercute também no setor específico dos resíduos (Diaz et al., 1997)

- A quase total inexistência de capacitação técnica, tanto de profissionais para desenvolvimento de estudos e pesquisas que vinculem ao gerenciamento dos resíduos sólidos as questões ambientais e de saúde, como de pessoal para operar os sistemas de limpeza urbana (Skinner, 1997);

- A prática de importação de tecnologia de países desenvolvidos sem a necessária adaptação às condições locais, que resulta, quase que inevitavelmente, em falhas e fracassos (Campbell, 1999; Wilson, 1995). São exemplos desta realidade: os equipamentos compactadores projetados para coleta de resíduos com baixo conteúdo de matéria orgânica úmida - nos Estados Unidos, a presença de restos de comida nos resíduos domiciliares varia entre $6 \%$ e $18 \%$ (Tchobanoglous et al., 1993), enquanto na América Latina varia entre $40 \%$ e $60 \%$ (Zepeda, 1995) - que propiciam o vazamento de líquidos ao longo das vias públicas, exalando mau cheiro e atraindo moscas; e as usinas de reciclagem e compostagem que, por razões semelhantes, operam com baixa eficiência (ou até mesmo não conseguem operar).

A Agenda 21 ressalta as dificuldades dos países em desenvolvimento de controlarem a poluição ambiental e adotarem medidas de proteção à saúde na mesma velocidade do desenvolvimento econômico (United Nations, 1992), cuja integração é essencial para garantir melhores padrões de qualidade de vida. Destaca ainda a importância de, em todos os casos, se levarem em conta os valores prevalentes em cada país e a extensão de aplicabilidade de padrões (de controle e tecnológicos) que são válidos para a maioria dos países desenvolvidos, mas que podem ser inadequados ou onerosos em demasia para os países em desenvolvimento.

A discussão das questões ambientais vem ocorrendo, no Brasil, de forma paralela ao desenvolvimento da saúde coletiva, sem a necessária articulação que possibilite experiências interdisciplinares como a complexidade da busca de soluções exige (Porto, 1998).

O objetivo do presente artigo é despertar a atenção para os problemas de saúde pública e saúde ocupacional relacionados ao (mau) gerenciamento dos resíduos sólidos, identificando as populações expostas e a origem dos riscos a que estão submetidas, procurando despertar o interesse dos pesquisadores da área de saúde, para que se avolumem pesquisas e estudos em busca de soluções. O artigo está baseado em uma revisão bibliográfica, na experiência dos autores, que atuam nas áreas de resíduos sólidos e saúde ocupacional há muitos anos, e também em alguns resultados de estudos recentes nos quais estiveram envolvidos.

\section{As populações expostas}

A principal dificuldade na definição das populações expostas aos efeitos diretos ou indiretos do gerenciamento inadequado dos resíduos sólidos municipais está no fato de os sistemas de informação e monitoramento sobre saúde e 
meio ambiente não contemplarem, em geral, o aspecto coletivo das populações, não dispondo de dados epidemiológicos suficientes e confiáveis. Existem poucos estudos epidemiológicos sobre a saúde dos trabalhadores dos sistemas de gerenciamento de resíduos sólidos municipais, mesmo nos países desenvolvidos (An et al., 1999). Apesar disso, algumas populações podem ser identificadas como suscetíveis de serem afetadas pelas questões ambientais, com redução da qualidade de vida e ampliação dos problemas de saúde.

A primeira população a ser considerada é aquela que não dispõe de coleta domiciliar regular e que, ao se desfazer dos resíduos produzidos, lançando-os no entorno da área em que vive, gera um meio ambiente deteriorado com a presença de fumaça, mau cheiro, vetores transmissores de doenças, animais que se alimentam dos restos, numa convivência promíscua e deletéria para a saúde (Ruberg \& Philippi Jr., 1999). Em geral, constituem esta população os segmentos pobres da sociedade. Entretanto, dependendo das condições e localização das suas moradias, os riscos se estendem às populações próximas, nem sempre de baixa renda, seja pelo alcance dos efeitos do mau cheiro e da fumaça, seja pela mobilidade dos vetores, como também pela invasão de áreas consideradas mais nobres, pelos resíduos arrastados por chuvas fortes, podendo propiciar condições favoráveis a epidemias de leptospirose e dengue, por exemplo.

Outra população sujeita à exposição aos resíduos municipais é a de moradores das vizinhanças das unidades de tratamento e destinação de tais resíduos. Por melhor que seja o padrão técnico da unidade - projeto, construção e operação - a questão do mau cheiro está sempre presente quando se manuseiam grandes quantidades de resíduos domiciliares, face ao processo de decomposição da matéria orgânica, e tem sido um dos fatores para o fechamento de usinas de reciclagem e compostagem no Brasil (Lua, 1999).

Na maioria das cidades da América Latina a situação se agrava pelo fato de os resíduos sólidos municipais serem dispostos no solo, de forma inadequada, em vazadouros a céu aberto (lixões). No Estado de São Paulo, por exemplo, 75\% dos municípios dispõem seus resíduos em lixões (CETESB, 1998). A necessidade de vias de melhor padrão, para acesso aos aterros e vazadouros, acaba por atrair moradores para as proximidades dos mesmos, sobretudo pela possibilidade do surgimento de linhas de ônibus, e essas pessoas constituem, em geral, uma população bastante exposta, pela total au- sência de saneamento básico. São populações que, além dos incômodos do mau cheiro, convivem com a presença de vetores e sofrem os efeitos da poluição/contaminação dos lençóis freáticos dos seus poços d'água, pelo chorume gerado nos vazadouros de lixo.

Ainda que seja difícil estabelecer uma relação de interferência na saúde desta população pelos resíduos sólidos municipais, diante da ausência de qualquer tipo de saneamento, não há razões para se imaginar que não haja uma contribuição significativa dos mesmos neste quadro negativo. Além disso, uma parcela desta população constitui os chamados catadores, que existem em praticamente todos os vazadouros de resíduos. Ao remexerem os resíduos vazados, à procura de materiais que possam ser comercializados ou servir de alimentos, os catadores estão expostos a todos os tipos de riscos de contaminação presentes nos resíduos, além dos riscos à sua integridade física por acidentes causados pelo manuseio dos mesmos e pela própria operação do vazadouro. Esta população, que normalmente vive próxima aos vazadouros, serve de vetor para a propagação de doenças originadas dos impactos dos resíduos, uma vez que parte da mesma trabalha em outras localidades, podendo transmitir doenças para pessoas com quem mantém contato.

Os impactos provocados pelos resíduos sólidos municipais podem estender-se para a população em geral, por meio da poluição e contaminação dos corpos d'água e dos lençóis subterrâneos, direta ou indiretamente, dependendo do uso da água e da absorção de material tóxico ou contaminado. A população em geral está ainda exposta ao consumo de carne de animais criados nos vazadouros e que podem ser causadores da transmissão de doenças ao ser humano. Estima-se que mais de cinco milhões de pessoas morrem por ano, no mundo inteiro, devido a enfermidades relacionadas com resíduos (Machado \& Prata Filho, 1999).

Os trabalhadores, diretamente envolvidos com os processos de manuseio, transporte e destinação final dos resíduos, formam outra população exposta. A exposição se dá notadamente: pelos riscos de acidentes de trabalho provocados pela ausência de treinamento, pela falta de condições adequadas de trabalho e pela inadequação da tecnologia utilizada à realidade dos países em desenvolvimento; e pelos riscos de contaminação pelo contato direto e mais próximo do instante da geração do resíduo, com maiores probabilidades da presença ativa de microorganismos infecciosos (An et al., 1999; Ferreira, 1997; Sivieri, 1995; Velloso et al., 1998). 
A adoção do modelo mundial de terceirização e privatização dos serviços de limpeza urbana nos países em desenvolvimento pode ter um reflexo negativo sobre a saúde dos trabalhadores, já que, além de uma redução nos seus padrões salariais, a rotatividade é extremamente elevada nas empresas privadas do setor, o que inviabiliza programas de treinamento e de prevenção. Isso deverá resultar em um aumento no número de acidentes e na deterioração dos, já baixos, padrões de saúde dos referidos trabalhadores.

\section{Efeitos na saúde humana e no meio ambiente}

Os mais freqüentes agentes físicos, químicos e biológicos presentes nos resíduos sólidos municipais e nos processos dos sistemas de seu gerenciamento, capazes de interferir na saúde humana e no meio ambiente são (Colombi et al., 1995; Ferreira, 1997; Velloso, 1995):

\section{Agentes físicos}

O odor emanado dos resíduos pode causar mal estar, cefaléias e náuseas em trabalhadores e pessoas que se encontrem proximamente a equipamentos de coleta ou de sistemas de manuseio, transporte e destinação final. Ruídos em excesso, durante as operações de gerenciamento dos resíduos, podem promover a perda parcial ou permanente da audição, cefaléia, tensão nervosa, estresse, hipertensão arterial. Um agente comum nas atividades com resíduos é a poeira, que pode ser responsável por desconforto e perda momentânea da visão, e por problemas respiratórios e pulmonares. Em algumas circunstâncias, a vibração de equipamentos (na coleta, por exemplo) pode provocar lombalgias e dores no corpo, além de estresse. Responsáveis por ferimentos e cortes nos trabalhadores da limpeza urbana, os objetos perfurantes e cortantes são sempre apontados entre os principais agentes de riscos nos resíduos sólidos.

Nem sempre lembrada, a questão estética é bastante importante, uma vez que a visão desagradável dos resíduos pode causar desconforto e náusea.

\section{Agentes químicos}

Nos resíduos sólidos municipais pode ser encontrada uma variedade muito grande de resíduos químicos, dentre os quais merecem destaque pela presença mais constante: pilhas e baterias; óleos e graxas; pesticidas/herbicidas; solventes; tintas; produtos de limpeza; cosméticos; remédios; aerossóis.

Uma significativa parcela destes resíduos é classificada como perigosa e pode ter efeitos deletérios à saúde humana e ao meio ambiente. Metais pesados como chumbo, cádmio e mercúrio, incorporam-se à cadeia biológica, têm efeito acumulativo e podem provocar diversas doenças como saturnismo e distúrbios no sistema nervoso, entre outras. Pesticidas e herbicidas têm elevada solubilidade em gorduras que, combinada com a solubilidade química em meio aquoso, pode levar à magnificação biológica e provocar intoxicações agudas no ser humano (são neurotóxicos), assim como efeitos crônicos (Kupchella \& Hyland, 1993).

\section{Agentes biológicos}

Os agentes biológicos presentes nos resíduos sólidos podem ser responsáveis pela transmissão direta e indireta de doenças.

Microorganismos patogênicos ocorrem nos resíduos sólidos municipais mediante a presença de lenços de papel, curativos, fraldas descartáveis, papel higiênico, absorventes, agulhas e seringas descartáveis e camisinhas, originados da população; dos resíduos de pequenas clínicas, farmácias e laboratórios e, na maioria dos casos, dos resíduos hospitalares, misturados aos resíduos domiciliares (Collins \& Kenedy, 1992; Ferreira, 1997).

Alguns agentes que podem ser ressaltados são: os agentes responsáveis por doenças do trato intestinal (Ascaris lumbricoides; Entamoeba coli; Schistosoma mansoni); o vírus causador da hepatite (principalmente do tipo B), pela sua capacidade de resistir em meio adverso; e o vírus causador da AIDS, mais pela comoção social que desperta do que pelo risco associado aos resíduos, já que apresenta baixíssima resistência em condições adversas. Além desses, devem também ser referidos os microorganismos responsáveis por dermatites.

A transmissão indireta se dá pelos vetores que encontram nos resíduos condições adequadas de sobrevivência e proliferação.

Entre os resíduos com presença de microorganismos, merecem ainda ser mencionados os resíduos infecciosos dos serviços de saúde que, pela falta de uma melhor compreensão dos modos de transmissão dos agentes associados a doenças infeciosas, têm sido alvo de receios exagerados da população em geral (Ferreira, 1997; Reinhardt et al., 1996; Rutala \& Mayhall, 1992). Contudo, isto não deve servir de justificativa para que as instituições de saúde não es- 
tabeleçam procedimentos gerenciais que reduzam os riscos associados a tais resíduos (principalmente dos perfurocortantes) com a sua desinfecção ou esterilização.

\section{Acidentes e riscos ocupacionais}

A saúde do trabalhador envolvido nos processos de operação do sistema de gerenciamento dos resíduos sólidos municipais está relacionada não só aos riscos ocupacionais inerentes aos processos, mas também às suas condições de vida (Anjos et al., 1995; Velloso, 1995).

Nos países latino-americanos não existem dados e informações sistematizados sobre acidentes de trabalho. Quanto a doenças relacionadas ao trabalho com resíduos sólidos municipais, as informações praticamente inexistem (Ferreira, 1997).

É claro que os riscos de acidentes e de agravos à saúde dependem da atividade exercida pelo trabalhador. Alguns dos acidentes mais freqüentes entre trabalhadores que manuseiam diretamente os resíduos sólidos municipais (Ferreira, 1997; Velloso et al., 1997) são descritos a seguir:

- Cortes com vidros: caracterizam o acidente mais comum entre trabalhadores da coleta domiciliar e das esteiras de catação de usinas de reciclagem e compostagem, e também entre os catadores dos vazadouros de lixo. As estatísticas deste tipo de acidente são subnotificadas, uma vez que os cortes de pequena gravidade não são, na maioria das vezes, informados pelos trabalhadores, que não os consideram acidentes de trabalho.

A principal causa destes acidentes é a falta de informação e conscientização da população em geral, que não se preocupa em isolar ou separar vidros quebrados dos resíduos apresentados à coleta domiciliar. A adoção obrigatória de sacos plásticos para o acondicionamento dos resíduos sólidos municipais, com efeitos positivos na qualidade dos serviços de limpeza urbana, infelizmente amplia os riscos pela opacidade dos mesmos e ausência de qualquer rigidez que possa proteger o trabalhador. A utilização de luvas pelo trabalhador atenua, mas não impede a maior parte dos acidentes, que não atingem apenas as mãos, mas também braços e pernas.

- Cortes e perfurações com outros objetos pontiagudos: espinhos, pregos, agulhas de seringas e espetos são responsáveis por corriqueiros acidentes envolvendo trabalhadores. Os motivos são semelhantes aos do item anterior.
- Queda do veículo: a natureza dos trabalhos no sistema de limpeza urbana, em especial na coleta domiciliar e operações especiais de limpeza de logradouros, acaba por obrigar o transporte dos trabalhadores nos mesmos veículos utilizados para a coleta e transporte dos resíduos. Isso faz com que as quedas de veículos sejam comuns. Dois aspectos são importantes como causas destes acidentes (muitos dos quais fatais): a inadequação dos veículos para tal transporte, onde o exemplo maior é o veículo de coleta em que os trabalhadores são transportados dependurados no estribo traseiro, sem nenhuma proteção (os veículos de coleta são construídos com base na tecnologia dos países desenvolvidos, onde a coleta é realizada por guarnições de no máximo dois homens, que viajam na cabine junto com o motorista); e a elevada presença de alcoolismo entre trabalhadores da limpeza urbana (Robazzi et al., 1992). - Atropelamentos: a eles estão expostos tanto os trabalhadores da coleta domiciliar e limpeza de logradouros como os trabalhadores de locais de transferência e destinação final dos resíduos. Além dos riscos inerentes à atividade, contribuem para os atropelamentos a sobrecarga e a velocidade de trabalho a que estão sujeitos os trabalhadores e o pouco respeito que os motoristas em geral têm para os limites e regras estabelecidas para o trânsito. Também deve ser lembrada a ausência de uniformes adequados (roupas visíveis, sapatos resistentes e antiderrapantes) como um fator de agravamento dos riscos de atropelamento.

- Outros: ferimentos e perdas de membros por prensagem em equipamentos de compactação e outras máquinas, mordidas de animais (cães, ratos) e picadas de formigas também fazem parte da relação de acidentes com resíduos sólidos municipais.

Especificar doenças ocupacionais relacionadas aos resíduos sólidos municipais é tarefa complexa. Os trabalhadores dos sistemas de limpeza urbana estão expostos a poeiras, a ruídos excessivos, ao frio, ao calor, à fumaça e ao monóxido de carbono, à adoção de posturas forçadas e incômodas e também a microorganismos patogênicos presentes nos resíduos municipais.

Além disso, entende-se que as condições de trabalho devam ser consideradas de "forma mais integrada e global, onde as cargas de trabalho são determinadas por fatores relativos ao processo de trabalho - a organização do trabalho e as condições ambientais; e por fatores relativos ao indivíduo - sexo, idade e condições de inserção na produção, nível de aprendizagem, condições de vida, estado de saúde físi- 
ca e emocional, motivação e interesse" (Mattos, 1992:11).

Um aspecto praticamente ainda não estudado é o da carga fisiológica de trabalho que as várias atividades desempenhadas pelos trabalhadores dos sistemas de limpeza urbana representam para o ser humano e o desgaste que podem provocar. A interação destes fatores com a exposição ocupacional pode aumentar o risco dos trabalhadores (Anjos \& Ferreira, 2000). Na coleta domiciliar, por exemplo, as características do processo de trabalho podem determinar hábitos alimentares bastante irregulares para os garis (tanto com relação ao horário como com relação à qualidade do alimento ingerido) que, associados a outros hábitos como o tabagismo e o consumo de álcool, podem ter efeitos deletérios à sua saúde, bem como ampliar os riscos de acidentes (Chor, 1999; Lima et al., 1997).

Com relação a doenças ocupacionais relacionadas às atividades com resíduos sólidos municipais, as micoses são comuns, aparecendo mais freqüentemente (mas não exclusivamente) nas mãos e pés, onde as luvas e calçados estabelecem condições favoráveis para o desenvolvimento de microorganismos.

Índices relativamente altos de doenças coronarianas e hipertensão arterial têm sido detectados entre trabalhadores da limpeza urbana (principalmente entre trabalhadores da coleta domiciliar). Cimino \& Mamtani (1987) encontraram $6,5 \%$ de hipertensão arterial e 2,2\% de doenças coronarianas; Anjos et al. (1995) encontraram $46 \%$ com algum grau de hipertensão arterial, dos quais $20 \%$ com sintomas moderados ou severos, nos trabalhadores da coleta domiciliar em uma gerência do Município do Rio de Janeiro. Apesar de maiores do que na população em geral, essas prevalências não comprovam uma relação definitiva de causa-efeito.

Em todas as operações, a exposição a poeiras orgânicas e microorganismos pode ser causadora de doenças do trato respiratório. Estudo realizado em uma planta de separação de resíduos na Dinamarca revelou que 53\% dos trabalhadores desenvolveram doença pulmonar durante os oito primeiros meses de produção (Malmros et al., 1992).

A exposição a acidentes com agulhas hipodérmicas e a eventual presença de microorganismos patogênicos podem ser responsáveis por acometimentos de hepatite B e AIDS entre outras doenças, nos trabalhadores. No entanto, não existem estudos que comprovem o nexo causal destas doenças nos trabalhadores envolvidos com os resíduos sólidos municipais.
Também deve-se fazer referência ao estresse, como resultado das tensões a que os trabalhadores estão sujeitos, dos longos períodos de transporte no trajeto casa-trabalho-casa, dos problemas de sobrevivência e agravos nutricionais (tanto desnutrição quanto obesidade) resultantes dos baixos salários e do desgaste que a carga fisiológica do trabalho pode produzir. O estresse pode ser a causa invisível de muitos dos acidentes de trabalho, pela redução da capacidade de autocontrole dos trabalhadores, e de doenças ocupacionais, pela redução das defesas naturais e do desgaste dos organismos.

\section{Conclusão}

Os problemas decorrentes dos resíduos sólidos municipais na América Latina continuam presentes e sem um equacionamento adequado. O lançamento indiscriminado dos resíduos no meio ambiente mantém-se como prática comum. Muitos dos vazadouros são à beira de cursos d'água (ou nos próprios), podendo provocar fortes impactos ambientais nos mesmos, rompendo o equilíbrio do ecossistema.

A presença dos resíduos sólidos municipais nas áreas urbanas é muito significativa, gerando problemas de ordem estética, de saúde pública, pelo acesso a vetores e animais domésticos, obstruindo rios, canais e redes de drenagem urbana, provocando inundações e potencializando epidemias de dengue e de leptospirose, entre outras.

Aos países em desenvolvimento não resta alternativa, senão a de uma mudança comportamental em relação aos resíduos, com redução na sua geração, utilização de tecnologias que estejam dentro das suas capacidades técnicas e de recursos, para gradativamente irem adquirindo maior controle sobre os efeitos ambientais e na saúde, provocados pelos seus próprios resíduos.

As medidas de prevenção e controle dos efeitos na saúde coletiva e na saúde ocupacional, dos resíduos sólidos municipais, dependem de informações e dados epidemiológicos em que sejam estabelecidos os nexos causais. $\mathrm{O}$ apoio a pesquisas dentro deste enfoque é prioritário.

O desenvolvimento de capacitação técnica, tendo em vista as questões ambientais e de saúde, dos profissionais envolvidos nos sistemas gerenciais de resíduos, poderá, a médio e longo prazo, introduzir estas variáveis nos projetos e planos.

A educação e conscientização da população em geral, sobre os efeitos ambientais e na 
saúde, da disposição inadequada dos seus resíduos e de suas responsabilidades enquanto cidadãos, exigirão um esforço muito grande, mas são básicos para uma mudança comportamental que irá repercutir diretamente no gerenciamento dos resíduos.

As condições básicas de vida a que todos os seres humanos têm direito (saúde, segurança, trabalho, educação, moradia etc.), dependem diretamente de um meio ambiente saudável
(Johnston, 1995). Os elevados índices de morbidade e mortalidade nos países em desenvolvimento, com os conhecimentos de prevenção que se têm, poderiam ser reduzidos quase aos níveis dos países desenvolvidos. As causas dos atuais excessos de doenças nos países em desenvolvimento são, na sua maioria, originárias do meio ambiente e poderiam essencialmente ser evitadas (Doll, 1992; Mendes, 1988).

\section{Referências}

ACCURIO, G.; ROSSIN, A.; TEIXEIRA, P. F. \& ZEPEDA, F., 1998. Diagnóstico de La Situación del Manejo de Residuos Sólidos Municipales en América Latina y El Caribe. Organización Panamericana de la Salud/Organización Mundial de La Salud, Serie Ambiental no 18. Washington, DC: Organización Panamericana de la Salud/Organización Mundial de La Salud.

AN, H.; ENGLEHARDT, J.; FLEMING, L. \& BEAN, J., 1999. Occupational health and safety amongst municipal solid waste workers in Florida. Waste Management \& Research, 17:369-377.

ANJOS, L. A.; BARROS, A. A.; FERREIRA, J. A.; OLIVEIRA, T. C. E.; SEVERINO, K. C.; SILVA, M. O. \& WAISSMANN, W., 1995. Gasto Energético e Carga Fisiológica de Trabalho em Coletores de Lixo Domiciliar no Rio de Janeiro: Um Estudo Piloto. Relatório de Pesquisa. Rio de Janeiro: Centro de Estudos da Saúde do Trabalhador e Ecologia Humana, Escola Nacional de Saúde Pública, Fundação Oswaldo Cruz.

ANJOS, L. A. \& FERREIRA, J. A., 2000. A avaliação da carga fisiológica de trabalho na legislação brasileira deve ser revista! O caso da coleta de lixo domiciliar. Cadernos de Saúde Pública, 16:785-790.

CAMPBELL, D. J. V., 1999. Institutional development for waste management in developing countries. Waste Management \& Research, 17:1-3.

CANTANHEDE, A., 1997. Experiences from the PanAmerican Centre of Sanitary Engineering \& Environmental Sciences - Difficulties and possibilities. In: Latin American-Swedish Seminar on Solid Waste Management, Proceedings, pp. 163-168. Rio de Janeiro: Associação Brasileira de Engenharia Sanitária e Ambiental/Lund University.
CETESB (Companhia de Tecnologia de Saneamento Ambiental), 1998. Inventário Estadual de Resíduos Sólidos Domiciliares. São Paulo: CETESB.

CHOR, D., 1999. Saúde Pública e mudanças de comportamento: Uma questão contemporânea. $\mathrm{Ca}$ dernos de Saúde Pública, 15:423-425.

CIMINO, J. A. \& MAMTANI, R., 1987. Occupational hazards for New York city sanitation workers. Journal of Environmental Health, 50:8-12.

COLLINS, C. H. \& KENEDY, D., 1992. The microbiological hazards of municipal and clinical wastes. Journal of Applied Bacteriology, 73:1-6.

COLOMBI, A.; BASILICO, S. \& FOÁ, V., 1995. Riesgos para la salud de los trabajadores asignados a las instalaciones de tratamiento y eliminacion de los desechos. Acta Toxicologica, 3:28-37.

DIAZ, L. F.; SAVAGE, G. M. \& EGGERTH, L. L., 1997. Managing solid wastes in developing countries. Wastes Management, 10:43-45.

DOLL, R., 1992. Health and the environment in the 1990s. American Journal of Public Health, 82:933940.

FERREIRA, J. A., 1997. Lixo Hospitalar e Domiciliar: Semelhanças e Diferenças - Estudo de Caso no Município do Rio de Janeiro. Tese de Doutorado, Rio de Janeiro: Escola Nacional de Saúde Pública, Fundação Oswaldo Cruz.

JOHNSTON, B. R., 1995. Human rights and the environment. Human Ecology, 23:111-123.

KUPCHELLA, C. D. \& HYLAND, M.C., 1993. Environmental Science - Living Within the System of $\mathrm{Na}$ ture. London: Prentice-Hall International.

LEITE, V. D. \& LOPES, W. S., 2000. Avaliação dos Aspectos Sociais, Econômicos e Ambientais Causados pelo Lixão da Cidade de Campina Grande. In: 
IX Simpósio Luso-Brasileiro de Engenharia Sanitária e Ambiental, Anais, CD-ROM IV. Porto Seguro: Associação Brasileira de Engenharia Sanitária e Ambiental.

LIMA, M. C.; DAMIÃO, J. J.; WERNERSBACH, L. \& ANJOS, L. A., 1997. Características nutricionais e fatores de risco para doenças cardiovasculares em coletores de lixo domiciliar no Município do Rio de Janeiro. In: 1o Congresso Brasileiro de Atividade Física e Saúde e 7o Simpósio de Pesquisa em Educação Física, Anais, p. 122. Florianópolis: Universidade Federal de Santa Catarina.

LUA, D., 1999. US\$50 milhões jogados no lixo. Jornal do Brasil, Rio de Janeiro, 12 dez. p. 17.

MACHADO, C. \& PRATA FILHO, D. A., 1999. Gestão de Resíduos Sólidos Urbanos em Niterói. In: 20으 Congresso Brasileiro de Engenharia Sanitária e Ambiental, Anais, CD-ROM III. Rio de Janeiro: Associação Brasileira de Engenharia Sanitária e Ambiental.

MAGLIO, I. C., 2000. Gestão Ambiental dos Resíduos Sólidos - O Papel dos Municípios. In: 9o Simpósio Luso-Brasileiro de Engenharia Sanitária e Ambiental, Anais, CD-ROM VI. Porto Seguro: Associação Brasileira de Engenharia Sanitária e Ambiental.

MATTOS, U. A. O., 1992. Introdução ao Estudo da Questão Saúde e Trabalho. Rio de Janeiro: Centro de Estudos da Saúde do Trabalhador e Ecologia Humana, Escola Nacional de Saúde Pública, Fundação Oswaldo Cruz.

MALMROS, P.; SIGSGAARD, T. \& BACH, B., 1992. Occupational Health Problems due to Garbage Sorting. Waste Management \& Research, 10:227-234.

MENDES, R., 1988. O Impacto dos efeitos da ocupação sobre a saúde dos trabalhadores. Revista de Saúde Pública, 22:311-326.

PORTO, M. F. S., 1998. Saúde, ambiente e desenvolvimento: Reflexões sobre a experiência da COPASAD - Conferência Panamericana de Saúde e Ambiente no Contexto de Desenvolvimento Sustentável. Ciência \& Saúde Coletiva, 3:33-46.

REINHARDT, P. A.; GORDON, J. \& ALVARADO, C. J. 1996. Medical waste management. In: Hospital Epidemiology and Infection Control (C. G. MayHall, ed.), pp. 1099-1108, 1st Ed., Baltimore: Williams \& Wilkins.

ROBAZZI, M. L. C.; MORIYA, T. M.; FÁVERO, M. \& PINTO, P. H. D., 1992. Algumas considerações sobre o trabalho dos coletores de lixo. Revista Brasileira de Saúde Ocupacional, 20:34-40.
RUBERG, C. \& PHILIPPI Jr., A., 1999. O Gerenciamento de Resíduos Sólidos Domiciliares: Problemas e Soluções - Um Estudo de Caso. In: 20o Congresso Brasileiro de Engenharia Sanitária e Ambiental, Anais, CD-ROM III. Rio de Janeiro: Associação Brasileira de Engenharia Sanitária e Ambiental.

RUTALA, W. A. \& MAYHALL, C. G., 1992. Medical waste. Infection Control and Hospital Epidemiology, 13:38-48.

SIVIERI, L. H., 1995. Saúde no trabalho e mapeamento de riscos. In: Saúde, Meio Ambiente e Condições de Trabalho - Conteúdos Básicos para uma Ação Sindical. São Paulo: Central Única dos Trabalhadores/Fundação Jorge Duprat Figueiredo de Segurança e Medicina no Trabalho.

SKINNER, J. H., 1997. Solid Waste Management - The Environmental Issue of the 90's. In: Latin American - Swedish Seminar on Solid Waste Management, Proceedings, pp. 115-118. Rio de Janeiro: Associação Brasileira de Engenharia Sanitária e Ambiental/Lund University.

TCHOBANOGLOUS, G.; THEISEN, H. \& VIGIL, S., 1993. Integrated Solid Waste Management Engineering Principles and Management Issues. Boston: Irwin/McGraw-Hill.

UNITED NATIONS, 1992. Drafts-Agenda 21 - Rio Declaration - Forest Principles. Rio de Janeiro: United Nations.

VELLOSO, M. P., 1995. Processo de Trabalho da Coleta de Lixo Domiciliar da Cidade do Rio de Janeiro: Percepção e Vivência dos Trabalhadores. Dissertação de Mestrado, Rio de Janeiro: Escola Nacional de Saúde Pública, Fundação Oswaldo Cruz.

VELLOSO, M. P.; SANTOS, E. M. \& ANJOS, L. A., 1997. Processo de trabalho e acidentes de trabalho em coletores de lixo domiciliar na cidade do Rio de Janeiro, Brasil. Cadernos de Saúde Pública, 13: 693-700.

VELLOSO, M. P.; VALLADARES, J. C. \& SANTOS, E. M. 1998. A coleta de lixo domiciliar na Cidade do Rio de Janeiro: Um estudo de caso baseado na percepção do trabalhador. Ciência \& Saúde Coletiva, 3:143-150.

WILSON, D. C., 1995. Waste Management in Developing Countries: Moving Towards the 21st Century. International Directory of Solid Waste Management 1994/95. The ISWA Yearbook. London: International Solid Waste Association.

ZEPEDA, F., 1995. El Manejo de Residuos Solidos Municipales En America Latina y El Caribe. Washington, DC: Organización Panamericana de la Salud. 